OPEN ACCESS

Edited by:

Neeraj Sharma,

University of New South Wales,

Australia

Reviewed by:

William Robert Brant,

Department of Chemistry, Uppsala

University, Sweden

Mogens Christensen

Aarhus University, Denmark

${ }^{*}$ Correspondence:

Marnix Wagemaker

m.wagemaker@tudelft.n

Specialty section: This article was submitted to

Energy Storage,

a section of the journa

Frontiers in Energy Research

Received: 04 April 2018

Accepted: 12 June 2018

Published: 03 July 2018

Citation:

Verhallen TW, LV S and Wagemaker M (2018) Operando Neutron Depth Profiling to Determine the Spatial Distribution of Li in Li-ion Batteries.

Front. Energy Res. 6:62. doi: 10.3389/fenrg.2018.00062

\section{Operando Neutron Depth Profiling to Determine the Spatial Distribution of Li in Li-ion Batteries}

\author{
Tomas W. Verhallen ${ }^{1}$, Shasha $L v^{2}$ and Marnix Wagemaker ${ }^{1 *}$ \\ ${ }^{1}$ Department of Radiation Science and Technology, Delft University of Technology, Delft, Netherlands, ${ }^{2}$ The State Key \\ Laboratory of New Ceramics and Fine Processing, School of Materials Science and Engineering, Tsinghua University, Beijing, \\ China
}

Neutron Depth Profiling (NDP) allows determination of the spatial distribution of specific isotopes, via neutron capture reactions. In a capture reaction charged particles with fixed kinetic energy are formed, where their energy loss through the material of interest can be used to provide the depth of the original isotope. As lithium- 6 has a relatively large probability for such a capture reaction, it can be used by battery scientists to study the lithium concentration in the electrodes even during battery operation. The selective measurement of the ${ }^{6} \mathrm{Li}$ isotope makes it a direct and sensitive technique, whereas the penetrative character of the neutrons allows practical battery pouch cells to be studied. Using NDP lithium diffusion and reaction rates can be studied operando as a function of depth, opening a large range of opportunities including the study of alloying reactions, metal plating, and (de) intercalation in insertion hosts. In the study of high rate cycling of intercalation materials the relatively low Li density challenges counting statistics while the limited change in electrode density due to the Li-ion insertion and extraction allows straightforward determination of the Li density as a function of electrode depth. If an electrode can be (dis)charged reversibly, data can be acquired and accumulated over multiple cycles to increase the time resolution. For Li metal plating and alloying reactions, the large lithium density allows good time resolution, however the large change of the electrode composition and density makes extracting the Li-density as a function of depth more challenging. Here an effective method is presented, using calibration measurements of the individual components, based on which the ratio of the components as a function of depth can be determined as well as the total Li-density. The same principles can be applied to insertion host materials, where the differences in density due to electrolyte infiltration yield the electrode porosity as a function of depth. This is of particular importance for battery electrodes where porosity has a direct influence on the energy density and charge transport.

Keywords: lithium-ion batteries, operando techniques, neutron depth profiling, electrode porosity, lithium concentration, spatial distribution, high rate materials, lithium metal plating 


\section{INTRODUCTION}

Neutron Depth Profiling (NDP), developed in 1972 (Ziegler et al., 1972), allows the detection of a small concentration of a certain isotope as function of sample depth, via neutron capture reactions (Downing et al., 1993). Applications range from boron impurity determination in silicon to boron nitrogen ratios in coatings (Ziegler et al., 1972; Downing et al., 1993; Ünlü et al., 1999). Whitney et al. (2009), first suggested using the ${ }^{6} \mathrm{Li}$ capture reaction for NDP on lithium ion battery systems. The technique offers the unique ability to quantitatively measure lithium concentrations, as function of depth, independent of oxidation state, during battery cycling (Oudenhoven et al., 2011; Wang et al., 2014; Harks et al., 2015; Liu and Co, 2016). Techniques allowing direct, non-invasive lithium detection operando are limited, as a result NDP was quickly adopted by a number of research groups in to order to study problems such as degradation (Nagpure et al., 2011) and charge transport limitations (Zhang et al., 2015). In the coming years the technique will become even more effective with the interest in light weight, high capacity anodes (Zuo et al., 2017), and cathodes, facilitating NDP measurements and the development of dedicated Li-battery setups (Çetiner et al., 2008; Tomandl et al., 2017). However, interpretation of the results will be more challenging as the electrode properties are dynamic, expanding, and contracting under the influence of lithium content (Mohanty et al., 2013; Liu et al., 2015; Liu and Co, 2016)

NDP exploits the neutron capture reaction of the lithium6 isotope, which is $7.5 \%$ abundant in natural lithium. Hence it measures ${ }^{6} \mathrm{Li}$ atoms only, regardless of their oxidation state, ensuring selectivity and low noise. The capture reaction is initiated by the absorption of a thermal neutron resulting in two charged particles with a well-defined energy: (Ziegler et al., 1972; Whitney et al., 2009).

$$
{ }^{6} \mathrm{Li}+n_{\text {thermal }} \stackrel{\text { yields }}{\longrightarrow} \mathrm{H}^{+}(2727.88 \mathrm{keV})+\mathrm{He}^{2+}(2055.12 \mathrm{keV})
$$

White beam thermal neutron fluxes at the Reactor Institute in Delft are in the order of $10^{7}$ neutrons $/ \mathrm{cm}^{2} / \mathrm{s}$ at the NDP the sample position. The neutron capture cross section of lithium- 6 is 940 barns (Kopecky, 1997) the absorption rate is one reaction per $10^{15} 6$-lithium atoms per second. This ${ }^{6} \mathrm{Li}$ consumption rate is negligible making NDP a non-destructive technique. Moreover as a white beam is used, no neutron optics are necessary, making the technique one of the most compact and cheapest amongst available experimental neutron set-ups (Utsuro and Ignatovich, 2010; Wang et al., 2012). A major advantage of using neutrons is the fact that they have a large penetration depth in materials, allowing relatively simple sample environments (Wang et al., 2012).

A typical set-up is depicted in Figure 1, adapted from Zhang et al. (2015). A charged particle detector is placed at a welldefined distance, thereby decreasing the opening angle such that particles arrive almost perpendicular to the detector plane, implying that the ${ }^{3} \mathrm{H}$ and $\mathrm{He}$ particle trajectories make $90^{\circ}$ with the sample plane. The detector measures the kinetic energy of the incoming helium and tritium particles, which has decreased because of interactions with the materials in the battery along their trajectory. The amount of material it encounters depends on the depth of the original Li; hence the energy loss is related to this depth. The energy loss of the ${ }^{3} \mathrm{H}$ and He particles, which depends on the composition and density of the battery electrode, is quantified by the stopping power. Therefore the stopping relates the measured energy loss to the depth position of Li. Using NDP a cross sectional averaged Li concentration profile is determined as a function of depth, the axis perpendicular to the sample surface (Whitney et al., 2009).

The stopping power depends on the density of charges, associated with the atoms, it is independent of the chemical nature of the surroundings, thus the stopping power of a mixture of materials is equal to the sum of volume ratio times the stopping power of the components (Ziegler, 1985; Ziegler et al., 2010):

$$
S_{t}=\sum_{i=1}^{n} v_{i} \cdot S_{i}
$$

Where $S$ represents the stopping power, $v$ the volume fraction and $i$ the different components, which in a Li-ion battery electrode would typically be carbon black, binder, electrolyte and active material. Accurate determination of the stopping power is paramount to achieve an accurate $\mathrm{Li}$ concentration profile and depth calibration. Electrons make up most of the matter volume and therefore the electron density dominates the stopping power, especially at high particle energies (Lindhard et al., 1963). At lower ${ }^{3} \mathrm{H}$ and He kinetic energies the type of bond of the electrons becomes relevant, where in the case of organic materials a correction factor of up to $20 \%$ is necessary to compensate for the decrease in electron energy with respect to the atomic state (Ziegler et al., 2010). Only at low particle kinetic energies do the atom cores start to contribute significantly to the stopping power (Wilson et al., 1977). As a consequence the stopping power is strongly dependent on the particle energy, being large for small kinetic energies and small for large kinetic energies. The consequence is that the depth resolution increases with increasing depth, assuming a constant energy resolution of the detector. This results in more counts per detector energy channel for larger energies and lower counting rates at lower energies, hence the standard error in the measurement is constant over the detector channels (Ziegler et al., 2010; Zhang et al., 2015). As the amount of detected particles is a function of energy accurate determination of the stopping power is necessary to achieve quantitative results from NDP, in addition to the geometric, background and detector corrections (Lindhard et al., 1963; Wilson et al., 1977; Ziegler, 1985; Maki et al., 1986; Ziegler et al., 2010).

Based on the well-defined kinetic energy of the ${ }^{3} \mathrm{H}$ and He particles after the capture reaction with ${ }^{6} \mathrm{Li}$, see Equation (1), there is a maximum depth from which the ${ }^{3} \mathrm{H}$ and $\mathrm{He}$ particles can exit the sample, which depends on the stopping power of the sample. Due to the larger charge of the (Ziegler et al., 2010; Zhang et al., 2015) ${ }^{4} \mathrm{He}^{2+}$ particle as compared 


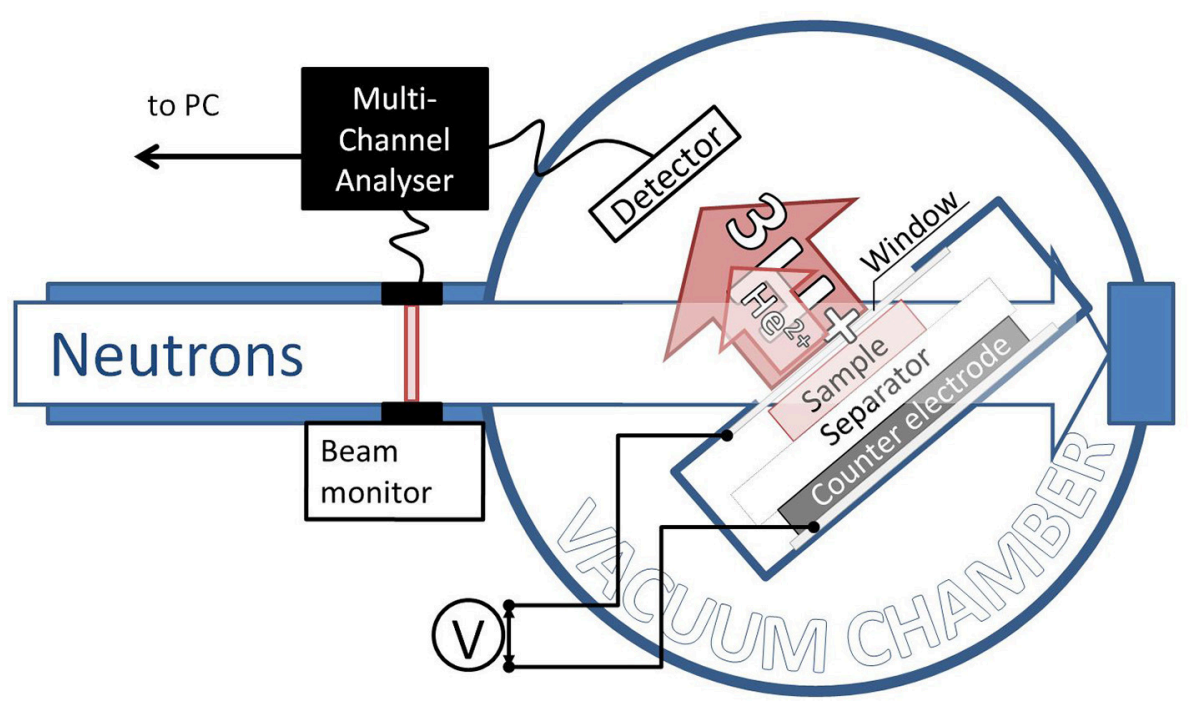

FIGURE 1 | Schematic setup of Neutron Depth Profiling.

to the ${ }^{3} \mathrm{H}^{+}$particle, the stopping power for ${ }^{4} \mathrm{He}^{2+}$ is larger, and consequentially the escape depth for ${ }^{4} \mathrm{He}^{2+}$ is smaller and the depth resolution is larger, making ${ }^{4} \mathrm{He}^{2+}$ suitable for the study of thin layers (Ziegler et al., 2010; Zhang et al., 2015). For energies smaller than the maximum energy of the ${ }^{4} \mathrm{He}^{2+}$ particle, $2056.12 \mathrm{keV}$, the measured ${ }^{3} \mathrm{H}$ and $\mathrm{He}$ signals overlap. Based on the ratio of the stopping power of the two particles, the two signals can be unraveled, methods for this can be found in Maki et al. (1986) and Liu et al. (2016). There is thus an intrinsic energy resolution associated with the material and particle through its stopping power, which through the detector resolution results in the depth resolution. For NDP the measurement time and the depth resolution are directly connected (Downing et al., 1993). Increasing the sample to detector distance, leads to higher resolution but fewer counts. However the relatively low beam intensity as compared to photon techniques is considered to be a common drawback of neutron techniques (Harks et al., 2015; Itkis et al., 2015). As a result, high resolution set-ups employ multiple detectors to improve counting statistics (Parikh et al., 1990; Mulligan et al., 2012). Another strategy to increase the count rate is to enrich the ${ }^{6} \mathrm{Li}$ contents of the materials under investigation (Downing et al., 1987; Oudenhoven et al., 2011) as the natural abundancy of the ${ }^{6} \mathrm{Li}$ isotope is only $7.5 \%$ (Hutchison, 1954). Hence replacing all ${ }^{7} \mathrm{Li}$ with ${ }^{6} \mathrm{Li}$ would increase count rates by a factor of 13.

The typical NDP operating conditions have implications for the operando/in situ battery research. An important implication of the geometrical resolution is that the detector is typically positioned at several centimeteres from the sample. This requires the setup to be operated under vacuum conditions to avoid parasitic energy absorption of the ${ }^{3} \mathrm{H}$ and $\mathrm{He}$ particles by the air (Hutchison, 1954; Downing et al., 1987; Oudenhoven et al., 2011). The vacuum conditions of the setup is one of the major obstacles for batteries containing liquid electrolytes, (Nagpure et al., 2014; Zhang et al., 2015) as most electrolytes contain solvents with a large vapor pressure, the evaporation of which leads to contact loss between the electrodes and the electrolyte, severely hindering battery performance (Zhang et al., 2015; Liu et al., 2016). To solve this Nagpure et al., have designed an experimental setup employing low density gasses at atmospheric pressures (Nagpure et al., 2014). Another approach is to work near the electrolyte vapor pressure, typically around 0.1 bar, allowing the particles to reach the detector with an acceptable loss in kinetic energy. Regardless of the operando set-up, a window is required to protect the unstable materials and supply electrons to the active material under study. This window is as thin as possible, reducing kinetic energy losses, while thick enough to withstand any pressure differences. For most cathode materials, aluminum is a straightforward choice (Zhang et al., 2015), as it is used in industry and provides low density and reasonable strength. For most anodes however, aluminum is not an option, enforcing the use of a metal/kapton combination for strength, conductivity and low density and thus stopping power (Liu D. X. et al., 2014; Wang C. et al., 2017).

Despite these challenges the technique is welcomed by the battery field, which is readily explained by the limited amount of techniques that are able to quantify both the amount and position of Li. Being a light element, lithium is difficult to detect with $\mathrm{X}$-rays, for which reason most photon techniques typically use the change in oxidation state of the heavier ions to observe lithium intercalation (Nonaka et al., 2006; Katayama et al., 2014) or changes in interatomic spacing upon lithium insertion or extraction (Ganapathy et al., 2014; Zhang et al., 2014). Neutrons probe atom cores and therefore favor detection of the lighter elements compared to X-rays (Itkis et al., 2015). Also the penetrative nature of 
neutrons and selectivity of the technique allows for probing small lithium concentrations in realistic battery pouch and coin cell set-ups without the introduction of noise (Whitney et al., 2009; Zhang et al., 2015; Vasileiadis et al., 2018). Furthermore the technique provides a unique relation between bulk properties, such as lithium concentration, and local submicron, depth dependent effects such as Ohmic potential drop or electrolyte concentration gradients (Zhang et al., 2015; Liu et al., 2016).

Previous operando NDP work has revealed a wealth of information. Oudenhoven et al. (2011)who were one of the first groups to perform operando NDP on a lithium ion cell, performed in situ lithium metal plating from a $\mathrm{LiCoO}_{2}$ cathode through a lipon solid electrolyte in a thin film system using the increased depth resolution of the ${ }^{4} \mathrm{He}^{2+}$ spectrum. Moreover by using different isotopes the authors demonstrated that lithium ion exchange between cathode and electrolyte is almost nonexistent in the pristine cell, due lack of lithium vacancies (Oudenhoven et al., 2011). It is noteworthy that the change in stopping power are marginal for Li-ion insertion hosts, making quantitative analysis straightforward. The first operando study on a liquid electrolyte cell was performed by Liu D. X. et al. (2014), their cell consisted of a 12.5 micron thick Sn anode. Their data clearly shows the expansion of the anode material, however the changes in the stopping power were not considered by the authors as the associated error in depth would stay within $10 \%$, which was deemed acceptable (Liu D. X. et al., 2014). In a later study, concerning the intercalation of lithium in $\mathrm{LiFePO}_{4}$, these problems where avoided as the changes in stopping power and morphology are negligible (Zhang et al., 2015). However, as typical for the low Li-density insertion hosts, the time resolution is poor, 10-60 min for operando (dis)charging (Zhang et al., 2015; Liu et al., 2016). Enriching samples with Li6 provides the opportunity to improve this, however it adds the complication of relaxation and homogenization effects if not all components are enriched (Oudenhoven et al., 2011). As shown recently,Li-metal plating is a process where full enrichment is achieved relatively easy (Lv et al., 2018). Furthermore, the intrinsically high lithium densities in these systems allow for a good time resolution, down to $60 \mathrm{~s}$. However, for these systems the challenge is correctly assessing the large changes in morphology for the calculation of the stopping power (Bieker et al., 2015; Liu et al., 2015; Cheng et al., 2017; Lv et al., 2018; Zhang, 2018). Challenges for Li-metal anodes include excessive growth of the solid electrolyte interface (SEI) and loss of active material, hindering its practical application (Zhang, 2018; Zhang et al., 2018). NDP is an ideal tool to study these problems but an effective strategy for determining the stopping power in these evolving electrode microstructures is paramount to obtain quantitative results.

At present two challenges are addressed in the operando study of batteries using NDP, aiming to demonstrate the possibilities for operando battery research. Firstly, building up statistics by repeated cycling is demonstrated as approach to improve the time resolution, making it possible to study Li-transport at high cycling rates, most relevant for insertion host electrode materials. Secondly, for electrodes that display large changes in morphology upon cycling, including Liair, Li-S, Li-metal, and alloying anodes, an approach for calculating the stopping power during operando experiments is demonstrated, making quantitative analysis possible (Wang et al., 2014; Kang et al., 2016; Asadi et al., 2018; Lv et al., 2018). Finally, the stopping power correction strategy brought forward makes it possible to determine the depth resolved porosity of electrodes, an important property for the charge transport in electrodes that is not straightforward by other techniques. By means of these examples the aim of the present work is to demonstrate the possibilities, measurement, and data analysis strategies relevant to operando NDP for Li-ion battery work.

\section{METHODS}

To illustrate the possibilities and stopping power strategies of operando NDP experiments three examples performed at the dedicated NDP beamline of the Reactor institute are reported and discussed. The set-up consists of an aluminum vacuum chamber where a Canberra PIPS detector is used to measure the energy of the emitted ${ }^{3} \mathrm{H}$ particles, as illustrated in Figure 1. All results are based on measurements on pouch cells (Gustafsson et al., 1992; Yu et al., 2006; Mohanty et al., 2013; Lv et al., 2018), also known as coffee bag cells (Singh et al., 2015), their flexible design allows the straightforward sealing of current collector window (Villevieille, 2015), as is also described in Zhang et al. (2014). Also pouch cell type constructions are common practice in industrial applications (Trask et al., 2014). Moreover reducing the amount of material is advisable as neutron activation can lead to samples emitting hazardous radiation. Pouch cells introduce negligible background by other isotopes as they consist from plastic coated aluminum foil.

The electrodes used are prepared by NMP based slurries cast onto 11 micron aluminum foil, in the first example a 90\% $\mathrm{Li}_{4} \mathrm{Ti}_{5} \mathrm{O}_{12}$ (Phostech), 3\% SuperP carbon and 7\% PVDF binder was used and in the third example, the double layer electrode with a first coating of $60 \% \mathrm{LiFePO}_{4}$ (Phostech) active material, $25 \%$ carbon species [of which $10 \%$ SuperP and 15\% KS4 (Timcal)] and 15\% PVDF binder and the second layer containing $80 \%$ of active material, $10 \%$ SuperP carbon and binder. The second layer was applied when the first was dry. Particle sizes of commercial powders are poorly defined, but XRD refinement showed an domain size of $\sim 140 \mathrm{~nm}$ for both the $\mathrm{LiFePO}_{4}$ and the $\mathrm{Li}_{4} \mathrm{Ti}_{5} \mathrm{O}_{12}$. (Singh et al., 2013a,b) In the second example bare 10 micron copper foil was used as current collector and window and ${ }^{6} \mathrm{Li}$-enriched Li-metal foil was used as counter electrode. $1 \mathrm{M} \mathrm{LiPF}_{6}$ in EC/DMC (Sigma Aldrich) served as the electrolyte in all examples. By measuring around the electrolyte vapor pressure in the NDP setup, contact loss between the electrodes of the pouch cell, signified by an increase in the overpotential, is avoided. The stopping power increases with decreasing particle energy, as a consequence conversion between energy and depth yields a nonlinear depth axis and a concentration profile that needs to 
be corrected in a subsequent step. A more direct method is to calculate the energy width (Equation 2.2) at a constant depth step (Equation 2.1) and then assign the corresponding counts (Equation 2.3) resulting in a linear depth axis and preserving the total counts.

$$
\begin{aligned}
d x & =x_{n+1}-x_{n} \\
E_{n+1} & =E_{n}-\frac{\partial E\left(E_{n}\right)}{\partial x} d x \\
C_{n} & =\int_{E_{n}}^{E_{n+1}} C(E) d E
\end{aligned}
$$

The step size, $d x$, is chosen, based on statistics and sample thickness. Depending on the stopping power the resolution for ${ }^{3} \mathrm{H}^{+}$can be around $50 \mathrm{~nm}$ and even better for $\mathrm{He}^{2+}$. However practical considerations such as counting rate and surface roughness generally compromise the resolution. A table containing stopping powers as a function of particle energy is obtained (Wang and Hong, 2007; Zhang et al., 2015; Liu and Co, 2016; Liu et al., 2016) using the open software package SRIM (Ziegler et al., 2010). Electrons associated in a molecular bond are delocalized and have significantly different binding energies. Hence to correct for materials with covalent bonds, such as the electrolyte, a compound correction factor is calculated (Ziegler et al., 2010). The resulting table shows the stopping power due to electrons as well as due to atom cores, the contributions are independent and can thus be summed, similar to mixtures. The approach for a two component system is similar to that described by Equations (2.1-2.3), the difference lies in an extra parameter $\rho$, used to define the ratio between the two components (electrolyte and Li metal for instance for Li-metal anodes);

$$
\begin{aligned}
d x & =x_{n+1}-x_{n} \\
E_{n+1} & =E_{n}-\left(\left.\rho_{n} \frac{\partial E\left(E_{n}\right)}{\partial x}\right|_{L i}+\left.\left(1-\rho_{n}\right) \frac{\partial E\left(E_{n}\right)}{\partial x}\right|_{E l}\right) d x \\
C_{n} & =\int_{E_{n}}^{E_{n+1}} C(E) d E
\end{aligned}
$$

The extra parameter results in an unsolvable set of equations, which can be resolved when the composition of the individual components is known, where:

$$
C_{n}=\rho_{n} C_{n, L i}+\left(1-\rho_{n}\right) C_{n, E l}
$$

Valid under the assumption that the measured concentration is solely due to the two components obtained previously. Similarly the depth dependent porosity can be deduced. As the amount of lithium contained within the electrolyte is considered negligible compared to pristine or discharged insertion hosts, the material quantity in both measurements, i.e., dry and filled with electrolyte is equal. Thus the integrated spectrum intensity is equal, and solely the stopping power is changed, spreading the signal. To find this addition in stopping power the equations must be solved in reverse order, starting from the counts obtained in the first measurement.

$$
\begin{aligned}
C_{n, d r y} & =C_{n, \text { filled }}=\left.\int_{E_{n}}^{E_{n+1}} C(E) d E\right|_{\text {filled }} \\
\Delta E_{E l} & =\left(E_{n+1}-E_{n}\right)_{\text {filled }}-\left(E_{n+1}-E_{n}\right)_{d r y} \\
d x_{n, \text { filled }} & =d x_{d r y}+\frac{\Delta E_{E l}}{\left.\frac{\partial E\left(E_{n}\right)}{\partial x}\right|_{E l}} \\
\rho_{n} & =1-\frac{d x_{d r y}}{d x_{n, f i l l e d}}
\end{aligned}
$$

Now due to the electrolyte filling the energy difference associated with the same amount of material, or counts, will be larger due to the larger stopping power, as is described by Equation (4.2). This energy difference is divided by the stopping power associated with the electrolyte, resulting in a pore length, or addition to the depth increment, Equation (4.3). The outcome of Equation (4.3) is a modified and non-linear depth axis, where the change in depth step reflects the local density.

\section{RESULTS AND DISCUSSION}

The possibilities of operando NDP, including the challenges in achieving good counting statistics and quantitative results through calculating the stopping power will be illustrated through three examples. Firstly, operando NDP of fast cycling $\mathrm{Li}_{4} \mathrm{Ti}_{5} \mathrm{O}_{12}$ electrodes is achieved, where the challenge is the counting statistics and where the limited change in stopping power due to the Li-ion insertion and extraction allows straightforward determination of the Li density as a function of electrode depth. Secondly, operando Li-metal plating is used to demonstrate the stopping power correction strategy necessary to achieve quantitative results for systems where the electrode composition changes strongly. At same time the large Li density results in high count rates and good time resolution during operando experiments. Thirdly, the stopping power calculation can also be used to quantify the change in stopping power of a porous Li-ion battery electrode due to filling with a Li containing electrolyte, providing the opportunity to determine the depth resolved porosity, demonstrated for $\mathrm{LiFePO}_{4}$ electrodes.

One of the major obstacles in the operando study of high rate intercalation materials with NDP is time resolution. First and foremost the number of detectors can be increased or the neutron intensity via focusing optics. If the electrode can be cycled reversibly another approach is to accumulate statistics over several cycles (Meija et al., 2016). Spinel $\mathrm{Li}_{4} \mathrm{Ti}_{5} \mathrm{O}_{12}$ (LTO) shows almost no strain upon (de)lithiation and its relatively high voltage prevents decomposition of typical Li-ion battery electrolytes (Tang et al., 2009; Odziomek et al., 2017; Wang S. et al., 2017). These properties ensure excellent rate capabilities, stable cycling and a long cycle life (Singh et al., 2013b). Previous measurements on another high rate material, $\mathrm{LiFePO}_{4}$ (Wang and Hong, 2007), revealed that at high currents, 20C, and larger (Liu et al., 2016), ionic conduction in the electrolyte is the limiting factor as indicated by the enormous differences in concentration and local current near the electrolyte compared 
to the current collector. Similar limitations may be expected to arise in LTO electrodes at comparable C-rates and particle sizes (Yu et al., 2015). However the limited time resolution restricts measurement possibilities at high C-rates.

Instead it is proposed to use the stable cycling of LTO and accumulate NDP measurements over several subsequent cycles at $30 \mathrm{C}, 0.0045 \mathrm{~A} / \mathrm{cm}^{2}$, and thereby achieve sufficient counting statistics in combination with the required time resolution $(30 \mathrm{~s}$ in this case). The stable cycling of the LTO electrode in the operando NDP cell is demonstrated in Figure 2A, displaying almost no change in the voltage characteristic during the 20 cycles, even at the reduced pressure condition required for operando NDP operation. Furthermore, denoted by the black squares, the change in state of charge, reflected in terms of occupied sites, as obtained from the NDP spectra measured every $30 \mathrm{~s}$, confirms the consistent behavior, reaching a site occupancy of 4.2-6.5 almost every time. The local, depth dependent, site occupancy or state of charge is found by comparing measurements to the lithium distribution after a low rate (dis)charge when all available active material is either filled, $\mathrm{Li}_{\mathrm{x}}=7$, or emptied, $\mathrm{Li}_{\mathrm{x}}=4$, completely. This allows the direct study of the active particle fraction as background noise due to the electrolyte lithium salt is subtracted. This method was previously described by Zhang et al. (2015).

Data obtained during these 20 charge-discharge cycles is ordered according to the voltage, where measurements within similar voltage ranges are averaged, reducing the noise, the result of which is shown in Figure 2B. The current collector is located on the left side at zero depth and the interface with the electrolyte at the right, approximately at 15 microns depth. Previous experiments observed large differences in concentration between the current collector and the interface with the electrolyte when $\mathrm{LiFePO}_{4}$ electrodes were charged with a 20C rate (Zhang et al., 2015; Liu et al., 2016). Close examination of Figure $\mathbf{2 B}$ and especially comparing electrolyte side and current collector side, reveals that there are almost no significant differences between these two regions of the electrode. The lithium concentration is in fact homogenously distributed throughout the electrode as opposed to that for $\mathrm{LiFePO}_{4}$ electrodes (by ex-situ measurements). This signifies the excellent rate performance of LTO electrodes, potentially explained by a better Li-ion transport network available through the particles (Lee et al., 2009; Tanida et al., 2016) or/and transport is facilitated due to a different morphology even though the particle size is comparable (Singh et al., 2013a,b). The particle size could also govern the kinetics, allowing a homogenous distribution since the larger particles do not have enough surface sites to accommodate larger reaction rates (Wang et al., 2016; Vasileiadis et al., 2018). The differences between the rate limiting mechanisms testifies to the extraordinary nature of these high rate insertion materials.

When electrode/electrolyte materials undergo changes during cycling, merging data over multiple cycles is not permitted and alternative strategies to improve the NDP measuring count rate are required. Decreasing the applied current might be the most straightforward strategy, alternatively the ${ }^{6} \mathrm{Li}$ concentration can be enriched or a neutron beam with a higher flux should be selected. A drawback is that unless all components have the same li- 6 concentration, some degree of homogenization (Oudenhoven et al., 2011) will occur during cycling, complicating quantitative data interpretation. In the study of lithium metal plating, this is straightforwardly resolved as there is no lithium containing electrode, reducing one ${ }^{7} \mathrm{Li}$ reservoir. Electrolyte enrichment is achieved by exposure to an abundance of ${ }^{6} \mathrm{Li}$ metal (96\% pure), before use in the operando NDP cell. For these specific measurements the use of ${ }^{6} \mathrm{Li}$ allows measurement times
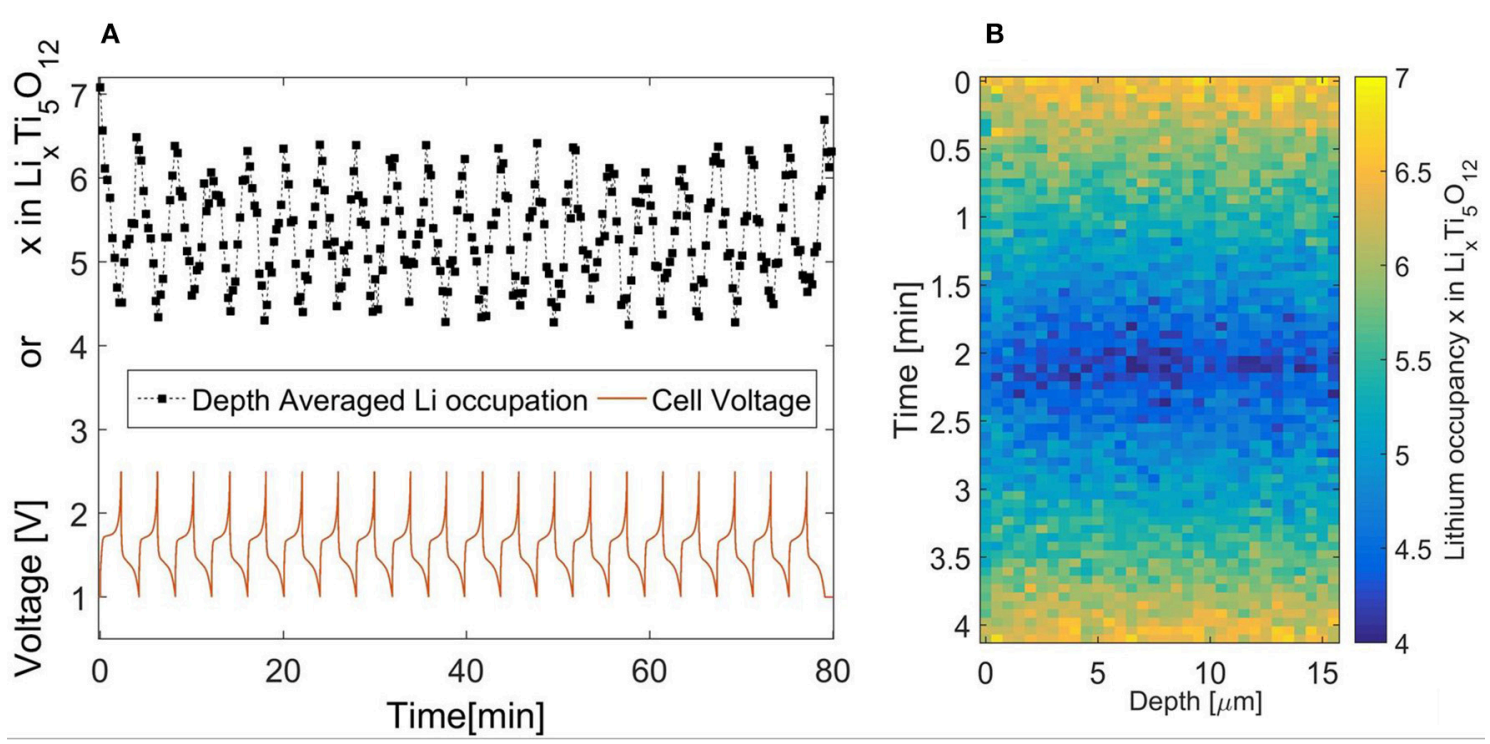

FIGURE 2 | (A) Reversible cycling of $\mathrm{Li}_{4} \mathrm{Ti}_{5} \mathrm{O}_{12}$ during operando NDP experiments at a $30 \mathrm{C}$ rate. (B) Operando NDP of LTO during $30 \mathrm{C}$ cycling, based on accumilated data over 20 cycles. The current collector at zero depth and the electrolyte/seperator at 15 micron depth. 
as short as $60 \mathrm{~s}$ (Lv et al., 2018). The challenge for these operando experiments is accurate calculation of the stopping power. Stopping powers of pure lithium and electrolyte are obtained straightforwardly using SRIM, shown in Figure 3. However determining the thickness and the concentration of the plated lithium layer requires knowledge of the ratio between the two components. Our proposed strategy is as follows, by obtaining spectra of both components, the local volume fraction of lithium and electrolyte can be fitted for any third measurement, as is described in Equations (3.1-3.4) found in methods. Obtaining the electrolyte spectra is straightforward as before cycling no lithium is plated and all counts are due to Li containing salts in the electrolyte.

Independently, NDP measurement of ${ }^{6} \mathrm{Li}$-metal is performed to provide the second component for the stopping power calculation. As ${ }^{6} \mathrm{Li}$ metal is a strong thermal neutron absorber the neutron beam intensity drops significantly along the sample thickness, (Figure 1), furthermore chances of recording a coincidence as a single event increase. The energy recorded will then equal the sum of the energy of the two particles, a detailed explanation of the implications for signal analysis can be found in the Supplementary Information. The decrease in neutron beam intensity will effectively result in a decreased measurement (Figure 1). This effectively results in a decreased measurement efficiency of the deeper parts of the sample as demonstrated in Figure 4A. The Li density for the uncorrected data, gray triangles, decreases as a function of depth, whereas for the Li metal it should be constant at $4.64 \times 10^{22}$ atoms per $\mathrm{cm}^{3}$, based on the ${ }^{6} \mathrm{Li}$ metal density $\left(0.353 \mathrm{~g} / \mathrm{cm}^{3}\right.$, Lord, 1992) and its molar weight $6.941 \mathrm{~g} / \mathrm{mol}$ (Meija et al., 2016). The first iteration, indicated by the black dots, corrects the count rate by the loss in neutron intensity based on the ${ }^{6} \mathrm{Li}$ cross-section of 940 barns (Kopecky, 1997). However, these atoms also absorb neutrons, thus a second iteration is necessary. Furthermore a small fraction is ${ }^{7} \mathrm{Li}$, hence the theoretical value, as is indicated by the dashed line in the

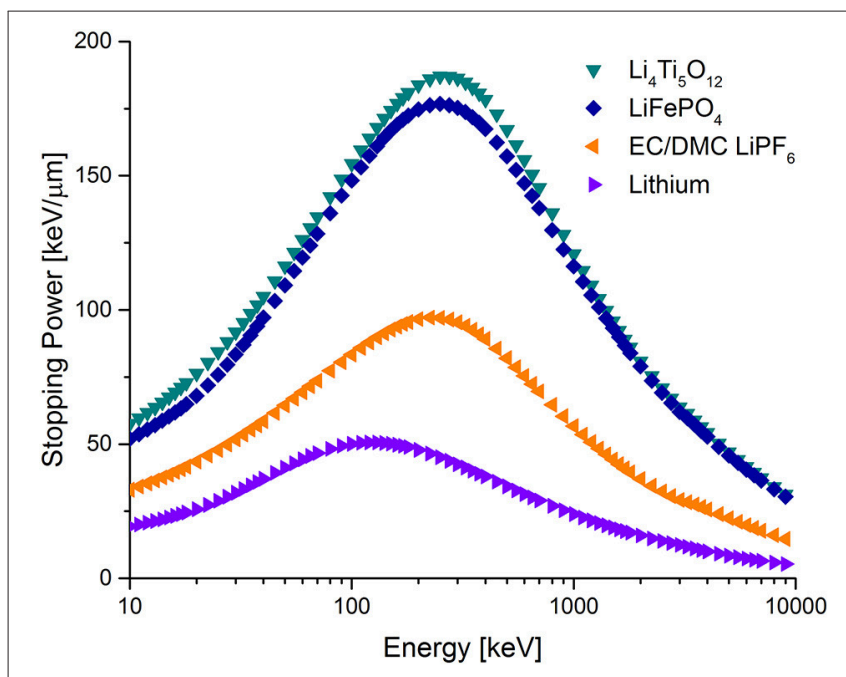

FIGURE 3 | Selected stopping powers. The transition metal insertion hosts have twice as high stopping compared the commonly used EC/DMC electrolyte, which in turn is almost twice as high as metallic lithium.
Figure $\mathbf{4 A}$, is obtained after the second iteration, indicated by the black squares, this results in the constant Li density, accounting for both ${ }^{6} \mathrm{Li}$ and ${ }^{7} \mathrm{Li}$.

The concentration increase at the interface with the $\mathrm{Cu}$ current collector should resemble a step function however as shown in Figure 4A, smooth increase is observed. Firstly, this is the consequence of the larger stopping power of the copper current collector/window, which pushes the signal to lower energies. Secondly, the lower stopping power of the lithium metal spreads the measurement inaccuracies across multiple microns.

In Figure 4B the ratio between Li-metal and electrolyte is shown at two moments during an operando Li-metal plating experiment. Firstly after a plating current of $1 \mathrm{~mA} / \mathrm{cm}^{2}$ for $1 \mathrm{~h}$, at this maximum capacity, only $30 \%$ of the volume is filled by Limetal. From this data, averaged over approximately $1 \mathrm{~cm}^{2}$ it is impossible to conclude if the Li-metal is plated as dense pillars or a porous network. SEM images, performed ex-situ on layers that were plated in the same electrolyte on bare copper, e.g., shown in Bieker et al. (2015) and Zhang et al. (2018), confirm that the electrochemically deposited Li-metal is highly porous. This porous layer will, to some extent, contain a solid electrolyte interphase, which is not taken into account in the stopping power analysis. The overall capacity as measured by NDP coincides with that applied electrochemically, indicating that no extra electrons are consumed by direct electrolysis of the electrolyte for instance. However it is difficult from NDP to obtain what fraction of Li is chemically decomposed. The lithium distribution after charge can be divided by the concentration after plating, as is shown in Figure 4C, providing an indication of the local charging efficiency and thus the ratio between lithium metal plated and inactive Li-metal/SEI layer formed. In the first few microns, on the left side near the current collector, there is a small plateau after which the ratio increases with increasing depth, indicating an increase of inactive material toward the electrolyte/separator on the right side. This indicates that plating results in dendrite like growing structures, whereas stripping induces thinning of these structures, resulting in the observed inactive layer covering the copper current collector. Although inactive $\mathrm{Li}$ in the SEI/Li metal morphology is not taken into account at present, the distribution and growth can be measured after each charge. These possibilities indicate that operando NDP can be used to providing vital experimental data on the distribution and growth at intermediate states of inactive Li (SEI and dead Li-metal) upon cycling of $\mathrm{Li}$ metal anodes.

In the lithium plating example discussed above, the stopping power of two known components was used in order to find the local ratio between the two during the operando NDP measurement. The same approach can be used to quantify the change in stopping power of a known porous Li-ion battery electrode due to filling with a Li containing electrolyte, providing the opportunity to determine the depth resolved porosity. In Figure 5A two ${ }^{3} \mathrm{H}$ count rates vs. particle kinetic energy loss are shown, one representing the dry electrode, in blue, and, in red, the same electrode impregnated by the electrolyte. The difference in NDP signal is due to the change in stopping power caused by the electrolyte filling the porous electrode. The data is corrected for the Al current collector, making direct comparison of the 


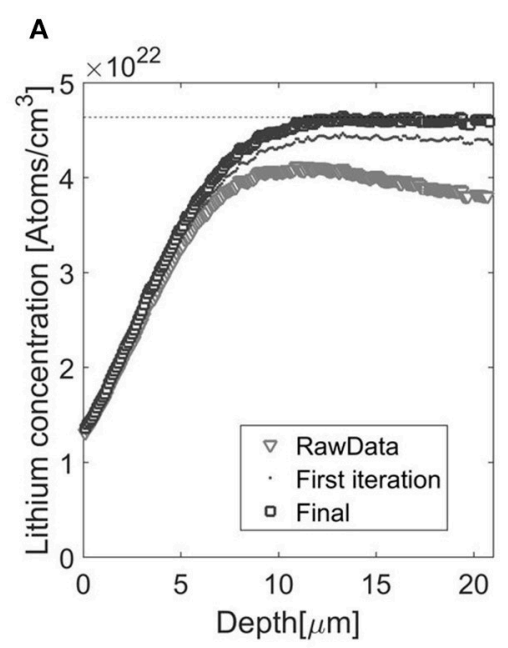

B

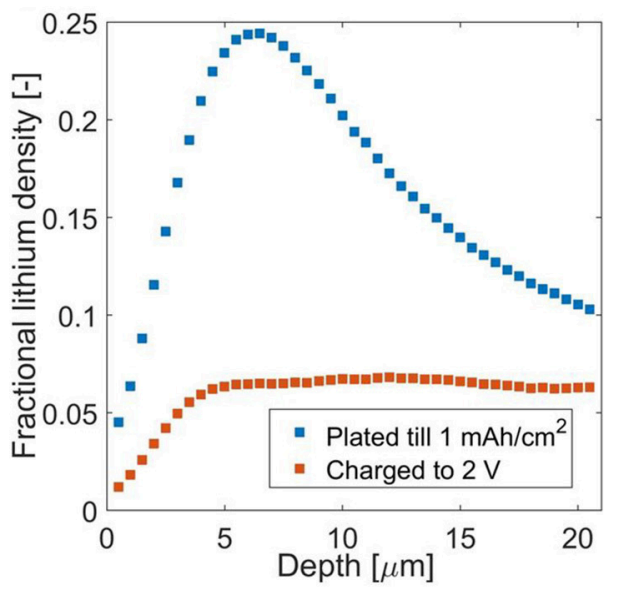

C

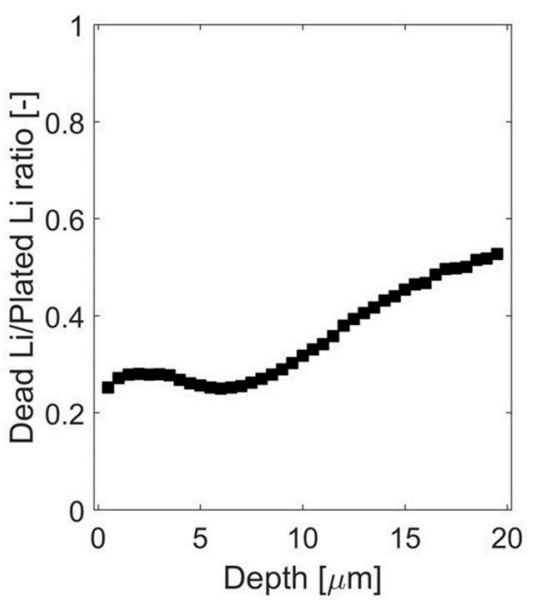

FIGURE 4 | (A) NDP spectra for lithium-6 metal foil, gray triangles indicate original, this data was corrected for reduced neutron intensity, dark gray dots, and finally for reduced neutron intensity and the small, unreactive ${ }^{7}$ li fraction, black squares. (B) Lithium concentration after plating (blue) and stripping (red) at $1 \mathrm{mAh} / \mathrm{cm}^{2}$ for $1 \mathrm{~h}$. The unit of vertical axis, fractional lithium density is defined as the observed Li-density devided by the Li-density of Li-metal. (C) Fraction of inactive Li, with respect to plated Li-metal with $1 \mathrm{mAh} / \mathrm{cm}^{2}$ for $1 \mathrm{~h}$ on copper.

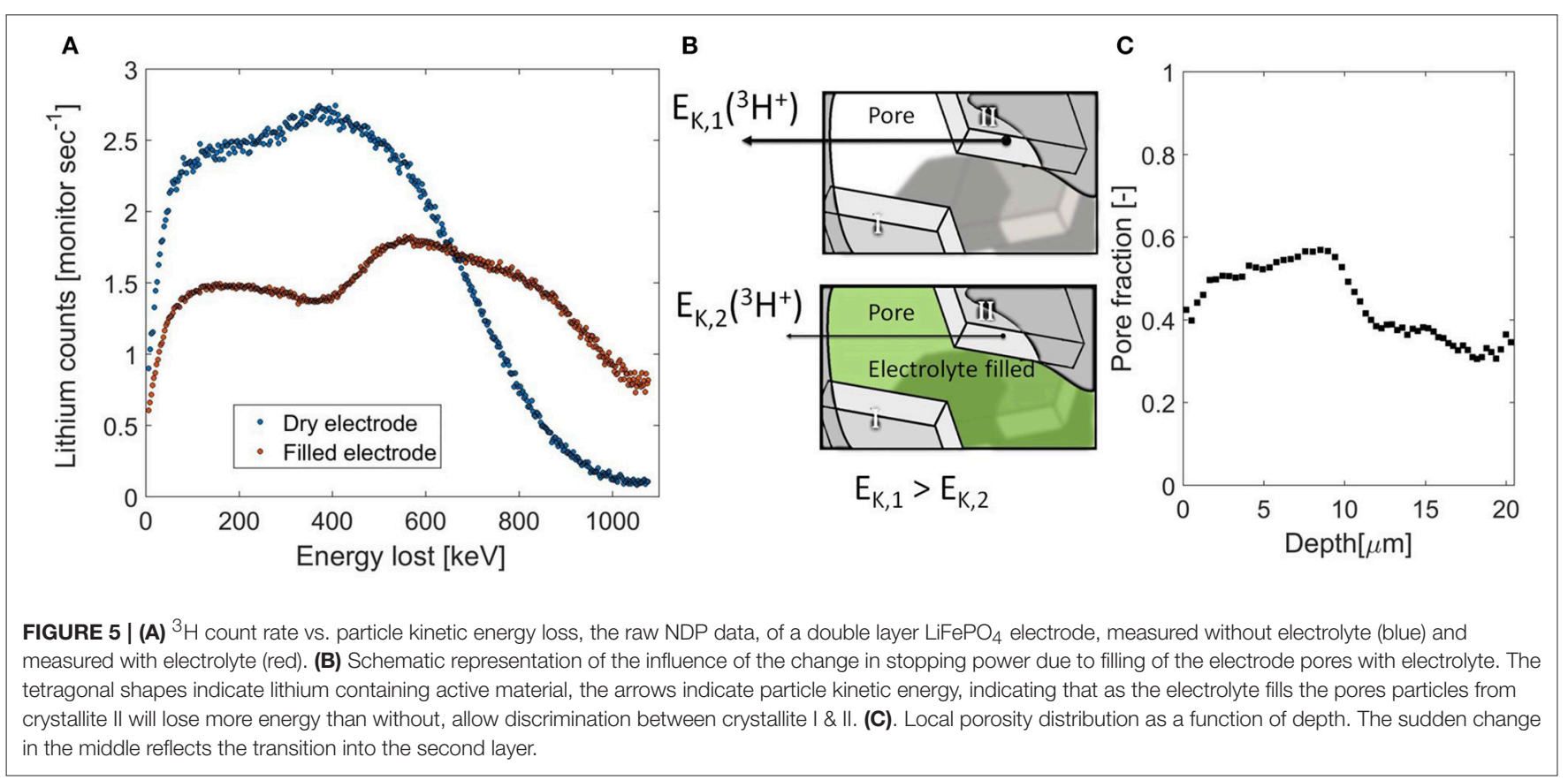

energy loss with and without the presence of the electrolyte possible. The electrode is built up by two layers of coating, the first layer on the $\mathrm{Al}$ current collector contains $60 \%$ of active material, 25\% carbon species, 15\% PVDF binder, and the second layer on top of that contains $80 \%$ of active material, $10 \%$ carbon, and binder. The two layers can be distinguished by NDP based on the difference in $\mathrm{Li}$ density, reflected in the step in ${ }^{3} \mathrm{H}$ count rate, especially when the electrode pores are filled with the electrolyte.

The electrolyte fills the pores in the electrode that would otherwise not contribute to the stopping power as schematically indicated in Figure 5B. Hence the stopping power is increased and the signal is spread across a larger number of energy channels, as is described in Equations (4.1-4.4). The total amount of lithium is only increased marginally $(\sim 5 \%)$ by adding the electrolyte (Zhang et al., 2015). Indeed the total amount of ${ }^{3} \mathrm{H}$ counts is equal and the increase in stopping power by adding the electrolyte results in a larger distribution in energy loss detected. This is a direct measure of the amount and distribution of the electrolyte through the electrode, from which the depth resolved pore filling fraction can be determined.

The result, shown in Figure 5C, indicates that the porosity is much higher in the first layer. This demonstrates that an increased carbon content also increases the porosity due to the 
large carbon black porosity. This readily provides an additional explanation why high carbon content electrodes exhibit high rate capabilities (Kang and Ceder, 2009; Liu H. et al., 2014). Also the porosity is relatively large at the vicinity of the interface between the two layers. This is probably a consequence of the double layer casting, where one layer is dried before the next is casted, resulting in more void space at the interface. For the second layer, having a closer to standard formulation of active material, carbon and binder, an average porosity of 0.36 is found, which is in good agreement with literature values (Singh et al., 2013a,b; Liu et al., 2016). Furthermore with this approach we measure the relevant porosity for the use as electrode porosity, the porosity that can be accessed by the electrolyte in question, which is different compared to what is measure by BET for instance (Klinkenberg, 1941). Electrode porosity is a crucial electrode parameter as it strongly influences battery performance (Fongy et al., 2010a,b; Strobridge et al., 2015; Just, 2016; Liu et al., 2017). Generally, a larger porosity favors Li-ion transport allowing larger (dis)charge (Singh et al., 2013b; Just, 2016) at the expense of the volumetric density (Liu et al., 2016; Singh et al., 2016) and electrical conductivity of the electrode (Wang and Hong, 2007; Fongy et al., 2010a). The optimum seems to be reached by a certain porosity gradient (Du et al., 2017; Liu et al., 2017). For measuring the porosity numerous methods are available, both bulk methods (DuBeshter et al., 2014; Li et al., 2014) as well as methods with a submicron resolution, such as tomography (Ge et al., 2014; Harks et al., 2015) and FIB-SEM (Hutzenlaub et al., 2014; Liu et al., 2016). The advantage of using NDP is the combination of both length scales, as this measurement represents an average over $1.1 \mathrm{~cm}^{2}$ electrode, the bulk average porosity is determined with a sub-micron resolution along an axis of interest. Moreover the measurement is performed in a non-destructive manner, allowing direct continuation of battery cycling, where the (de)lithiation as a function of depth and local porosity can now be studied.

\section{CONCLUSION}

Direct observation of $\mathrm{Li}$ concentration vs. electrode depth in working Li-ion batteries is possible with NDP through the capture reaction of the thermal neutrons by the ${ }^{6} \mathrm{Li}$ isotope. Although the large attenuation length of neutrons in most materials allows diverse sample environments, the relatively low neutron intensity requires smart experimental design. The translation toward quantitative results requires detailed consideration of the stopping power of the battery electrodes ${ }^{6} \mathrm{Li}$ enrichment is an effective solution but not always practical to achieve. Here we demonstrate that accumulation of NDP data over repeated battery cycling enables operando investigations of intercalation materials at a rate of $30 \mathrm{C}$, avoiding complexity of ${ }^{6} \mathrm{Li}$ homogenization that comes along with enrichment and allowing for the use of commercially produced powders.

The charged particles formed in the neutron capture reaction during NDP measurements allow straightforward detection of $\mathrm{Li}$, where their energy loss is a direct measure for the point of origin and hence for the depth. Their kinetic energy limits the escape depth and therefore the depth of view, which demands smart design of the battery window and current collector, in particular in the low pressure NDP environment. Accurate determination of the stopping power of the battery electrode, which may change upon cycling, is vital to achieve the quantitative $\mathrm{Li}$ density as a function of depth. In particular for the cases that electrochemical cycling leads to large changes in the composition, a stopping power calculation strategy is developed to result in quantitative results. This is demonstrated lithium metal plating, where operando NDP is able to provide insight in the growth and development of the evolution of inactive Li.

Furthermore it is demonstrated that the change in NDP signal due to filling of the porous electrode by the electrolyte can be used to determine the depth resolved filling fraction or porosity. This is in particular important for battery electrodes where porosity has a direct influence on the energy density and charge transport, hence determining the rate capability of battery electrodes.

\section{AUTHOR CONTRIBUTIONS}

TV and MW wrote the manuscript. TV developed data treatment methods. Designed and conducted the experiments used in example 1 \& 3 using intercalation materials, concerning $\mathrm{Li}_{4} \mathrm{Ti}_{5} \mathrm{O}_{12}$ and the porosity determination. SL conducted the lithium plating experiment.

\section{FUNDING}

The research leading to these results has received funding from the European Research Council under the European Union's Seventh Framework Programme FP/2007-2013)/ERC Grant Agreement No. [307161] of MW Financial support from the Advanced Dutch Energy Materials (ADEM) program of the Dutch Ministry of Economic Affairs, Agriculture and Innovation is gratefully acknowledged. Financial support of the KNAW for the joint research project under the scientific cooperation between China and the Netherlands, project number $530-5 \mathrm{CDP} 10$ is gratefully acknowledged. SL is grateful for the financial support by National Key R\&D Plan of China (No. 2017YFB0702201).

\section{ACKNOWLEDGMENTS}

The technical staff at the Reactor Institute Delft (RID) is gratefully acknowledged for their all-round support, most notable Michel Steenvoorden and Freek Labohm for maintaining the NDP set-up running and Frans Ooms for the pouch cell facilities.

\section{SUPPLEMENTARY MATERIAL}

The Supplementary Material for this article can be found online at: https://www.frontiersin.org/articles/10.3389/fenrg. 2018.00062/full\#supplementary-material 


\section{REFERENCES}

Asadi, M., Sayahpour, B., Abbasi, P., Ngo, A. T., Karis, K., et al. (2018). A lithiumoxygen battery with a long cycle life in an air-like atmosphere. Nature 555:502. doi: 10.1038 /nature25984

Bieker, G., Winter, M., and Bieker, P. (2015). Electrochemical in situ investigations of SEI and dendrite formation on the lithium metal anode. Phys. Chem. Chem. Phys. 17, 8670-8679. doi: 10.1039/C4CP05865H

Çetiner, S. M., Ünlü, K., and Downing, R. G. (2008). Development and applications of time-of-flight neutron depth profiling (TOF-NDP). J. Radioanal. Nuclear Chem. 276:623. doi: 10.1007/s10967-008-0609-7

Cheng, X.-B., Zhang, R., C.-,Zhao, Z., and Zhang, Q. (2017). Toward safe lithium metal anode in rechargeable batteries: a review. Chem. Rev. 117, 10403-10473. doi: 10.1021/acs.chemrev.7b00115

Downing, R. G., Lamaze, G. P., Langland, J. K., and Hwang, S. T. (1993). Neutron depth profiling: overview and description of NIST facilities. J. Res. Natl. Instit. Stand. Technol. 98, 109-126. doi: 10.6028/jres.098.008

Downing, R., Maki, J., and Fleming, R. (1987). Analytical applications of neutron depth profiling. J. Radioanal. Nucl. Chem. 112, 33-46. doi: 10.1007/BF02037274

Du, Z., Wood, D. L., Daniel, C., Kalnaus, S., and Li, J. (2017). Understanding limiting factors in thick electrode performance as applied to high energy density Li-ion batteries. J. Appl. Electrochem. 47, 405-415. doi: 10.1007/s10800-017-1047-4

DuBeshter, T., Sinha, P. K., Sakars, A., Fly, G. W., and Jorne, J. (2014). Measurement of tortuosity and porosity of porous battery electrodes. J. Electrochem. Soc. 161, A599-A605. doi: 10.1149/2.073404jes

Fongy, C., Gaillot, A. C., Jouanneau, S., Guyomard, D., and Lestriez, B. (2010a). Ionic vs electronic power limitations and analysis of the fraction of wired grains in LiFePO4 composite electrodes. J. Electrochem. Soc. 157, A885-A891. doi: 10.1149/1.3432559

Fongy, C., Jouanneau, S., Guyomard, D., Badot, J. C., and Lestriez, B. (2010b). Electronic and ionic wirings versus the insertion reaction contributions to the polarization in LiFePO4 composite electrodes. J. Electrochem. Soc. 157, A1347-A1353. doi: 10.1149/1.3497353

Ganapathy, S., Adams, B. D., Stenou, G., Anastasaki, M. S., Goubitz, K. X., et al. (2014). Nature of $\mathrm{Li} 2 \mathrm{O} 2$ oxidation in a $\mathrm{Li}-\mathrm{O} 2$ battery revealed by operando x-ray diffraction. J. Am. Chem. Soc. 136, 16335-16344. doi: 10.1021/ja508794r

Ge, M., Lu, Y., Ercius, P., Rong, J., Fang, X., Mecklenburg, M., et al. (2014). Large-scale fabrication, 3D tomography, and lithium-ion battery application of porous silicon. Nano Lett. 14, 261-268. doi: 10.1021/nl403923s

Gustafsson, T., Thomas, J. O., Koksbang, R., and Farrington, G. C. (1992). The polymer battery as an environment for in situ X-ray diffraction studies of solid-state electrochemical processes. Electrochimica Acta 37, 1639-1643. doi: 10.1016/0013-4686(92)80128-9

Harks, P. P., Mulder, F. M., and Notten, P. H. L. (2015). In situ methods for Li-ion battery research: a review of recent developments. J. Power Sourc. 288, 92-105. doi: 10.1016/j.jpowsour.2015.04.084

Hutchison, D. A. (1954). Natural abundance of the lithium isotopes. Phys. Rev. 96, 1018-1021. doi: 10.1103/PhysRev.96.1018

Hutzenlaub, T., Thiele, S., Paust, N., Spotnitz, R., Zengerle, R., and Walchshofer, C. (2014). Three-dimensional electrochemical Li-ion battery modelling featuring a focused ion-beam/scanning electron microscopy based threephase reconstruction of a LiCoO2 cathode. Electrochimica Acta 115, 131-139. doi: 10.1016/j.electacta.2013.10.103

Itkis, M. D., Velasco Vélez, J., Knop-Gericke, A., Vyalikh, A. V., Avdeev, M., and Yashina, L. (2015). Probing operating electrochemical interfaces by photons and neutrons. ChemElectroChem 2, 1427-1445. doi: 10.1002/celc.2015 00155

Just, P. (2016). A method to quantify coating thickness and porosity of electrodes for lithium-ion-batteries. Measurement 89:312. doi: 10.1016/j.measurement.2016.04.001

Kang, B., and Ceder, G. (2009). Battery materials for ultrafast charging and discharging. Nature 458:190. doi: 10.1038/nature07853

Kang, H. S., Park, E., Hwang, J., Y., Kim, H., Aurbach, D., et al. (2016). A scaled-up lithium (ion)-sulfur battery: newly faced problems and solutions. Adv. Mater. Technol. 1:1600052. doi: 10.1002/admt.201600052

Katayama, M., Sumiwaka, K., Miyahara, R., Yamashige, H., Arai, H., Uchimoto, Y., et al. (2014). X-ray absorption fine structure imaging of inhomogeneous electrode reaction in LiFePO4 lithium-ion battery cathode. J. Power Sourc. 269, 994-999. doi: 10.1016/j.jpowsour.2014.03.066

Klinkenberg, L. J. (1941). The Permeability Of Porous Media To Liquids And Gases. Drilling and Production Practice. New York, NY, American Petroleum Institute.

Kopecky, J. (1997). Atlas of Neutron Capture Crossections. Vienna:IAEA Nuclear Data Section.

Lee, K. T., Kan, W. H., and Nazar, L. F. (2009). Proof of intercrystallite ionic transport in LiMPO4 electrodes $(\mathrm{M}=\mathrm{Fe}, \mathrm{Mn})$. J. Am. Chem. Society 131, 6044-6045. doi: 10.1021/ja8090559

Li, X., Gu, M., Hu, S., Kennard, R., Yan, P., Chen, X., et al. (2014). Mesoporous silicon sponge as an anti-pulverization structure for high-performance lithiumion battery anodes. Nat. Commun. 5:4105. doi: 10.1038/ncomms5105

Lindhard, J., Scharff, M., and Schioett, H. E. (1963). Range concepts and heavy ion ranges (notes on atomic collisions, II). Kgl. Danske Videnskab. Selskab. Mat. Fys. Medd. 33, 1-42.

Liu, D. X., and Co, A., C. (2016). Revealing chemical processes involved in electrochemical (De)lithiation of $\mathrm{Al}$ with in situ neutron depth profiling and X-ray diffraction. J. Am. Chem. Soc. 138, 231-238. doi: 10.1021/jacs.5b10295

Liu, D. X., Cao, L. R., and Co, A. C. (2015). In Situ Neutron Depth Profiling of Lithium Transport Within Aluminum and Tin. Meeting Abstracts MA201501(2), 649.

Liu, D. X., Wang, J., Pan, K., Qiu, J., Canova, M., Cao, L., et al. (2014). In situ quantification and visualization of lithium transport with neutrons. Angew. Chem. Internat. Editn 53, 9498-9502. doi: 10.1002/anie.201404197

Liu, H., Strobridge, F. C., Borkiewicz, O. J., Wiaderek, K. M., Chapman, K. W., et al. (2014). Capturing metastable structures during high-rate cycling of $\mathrm{LiFePO}<\mathrm{sub}>4</$ sub $>$ nanoparticle electrodes. Science 344:6191. doi: $10.1126 /$ science. 1252817

Liu, L., Guan, P., and Liu, C. (2017). Experimental and simulation investigations of porosity graded cathodes in mitigating battery degradation of high voltage lithium-ion batteries. J. Electrochem. Soc. 164, A3163-A3173. doi: $10.1149 / 2.1021713$ jes

Liu, Z., Verhallen, T. W., Singh, D. P., Wang, H. Q., Wagemaker, M., and Barnett, S. (2016). Relating the 3D electrode morphology to Li-ion battery performance; a case for LiFePO4. J. Power Sourc. 324, 358-367. doi: 10.1016/j.jpowsour.2016.05.097

Lord, A. M. J., (1992). Macmillan's Chemical and Physical Data. London: Macmillan.

Lv, S., Verhallen, T., Vasileiadis, A., Ooms, F., Xu, Y., Li, Z., et al. (2018). Operando monitoring the Lithium spatial distribution of Lithium metal anodes. Nat. Commun. 9:2152. doi: 10.1038/s41467-018-04394-3

Maki, J. T., Fleming, R. F., and Vincent, D. H. (1986). Deconvolution of neutron depth profiling spectra. Nucl. Instr. Methods Phys. Res. Sec. B 17, 147-155. doi: 10.1016/0168-583X(86)90077-7

Meija, J., Coplen Tyler, B., Berglund, M., Brand Willi, A., De Bièvre, P., Gröning, M., et al. (2016). Atomic weights of the elements 2013 (IUPAC Technical Report). Pure Appl. Chem. 88:265. doi: 10.1515/pac-2015-0305

Mohanty, D., Kalnaus, S., Meisner, R. A., Rhodes, K. J., Li, J., et al. (2013). Structural transformation of a lithium-rich Li1.2Co0.1Mn0.55Ni0.15O2 cathode during high voltage cycling resolved by in situ X-ray diffraction. J. Power Sourc. 229, 239-248. doi: 10.1016/j.jpowsour.2012.11.144

Mulligan, P. L., Cao, L. R., and Turkoglu, D. (2012). A multi-detector, digitizer based neutron depth profiling device for characterizing thin film materials. Rev. Sci. Instr. 83:8. doi: 10.1063/1.4732168

Nagpure, S. C., Downing, R. G., Bhushan, B., Babu, S. S., and Cao, L. (2011). Neutron depth profiling technique for studying aging in Li-ion batteries. Electrochim. Acta 56, 4735-4743. doi: 10.1016/j.electacta.2011.02.037

Nagpure, S. C., Mulligan, P., Canova, M., and Cao, L. R. (2014). Neutron depth profiling of Li-ion cell electrodes with a gas-controlled environment. J. Power Sourc. 248, 489-497. doi: 10.1016/j.jpowsour.2013.09.032

Nonaka, T., Okuda, C., Seno, Y., Nakano, H., Koumoto, K., and Ukyo, Y. (2006). In situ XAFS and micro-XAFS studies on LiNi0.8Co0.15Al0.05O2 cathode material for lithium-ion batteries. J. Power Sourc. 162, 1329-1335. doi: 10.1016/j.jpowsour.2006.09.003

Odziomek, M., Chaput, F., Rutkowska, A., Swierczek, K., Olszewska, D., Sitarz, M., et al. (2017). Hierarchically structured lithium titanate for ultrafast charging in long-life high capacity batteries. Nat. Commun. 8:15636. doi: $10.1038 /$ ncomms 15636 
Oudenhoven, J. F. M., Labohm, F., Mulder, M., Niessen, R. A. H., Mulder, F., M., and Notten, P. H. L. (2011). In situ neutron depth profiling: a powerful method to probe lithium transport in micro-batteries. Adv. Mater. 23:4103. doi: 10.1002/adma.201101819

Parikh, N. R., Frey, E. C., Hofsäss, H. C., Swanson, M. L., Downing, R. G., et al. (1990). Neutron depth profiling by coincidence spectrometry. Nucl. Instr. Methods Phys. Res. Sec. B 45, 70-74. doi: 10.1016/0168-583X(90)90787-U

Singh, D. P., Mulder, F. M., Abdelkader, A. M., and Wagemaker, M. (2013a). Facile micro templating $\mathrm{LiFePO} 4$ electrodes for high performance Li-Ion batteries. Adv. Energy Mater. 3, 572-578. doi: 10.1002/aenm.201200704

Singh, D. P., Mulder, F. M., and Wagemaker, M. (2013b). Templated spinel Li4Ti5O12 Li-ion battery electrodes combining high rates with high energy density. Electrochem. Commun. 35, 124-127. doi: 10.1016/j.elecom.2013.08.014

Singh, M., Kaiser, J., and Hahn, H. (2015). Thick electrodes for high energy lithium ion batteries. J. Electrochem. Soc. 162, A1196-A1201. doi: 10.1149/2.0401507jes

Singh, M., Kaiser, J., and Hahn, H. (2016). Effect of porosity on the thick electrodes for high energy density lithium ion batteries for stationary applications. Batteries 2:35. doi: 10.3390/batteries 2040035

Strobridge, F. C., Orvananos, B., Croft, M. H.,Yu, C., Robert, R., et al. (2015). Mapping the inhomogeneous electrochemical reaction through porous LiFePO4-electrodes in a standard coin cell battery. Chem. Mater. 27, 2374-2386. doi: 10.1021/cm504317a

Tang, Y., Yang, L., Fang, S., and Qiu, Z. (2009). Li4Ti5O12 hollow microspheres assembled by nanosheets as an anode material for high-rate lithium ion batteries. Electrochim. Acta 54, 6244-6249. doi: 10.1016/j.electacta.2009.05.092

Tanida, H., Yamashige, H., Orikasa, Y., Gogyo, Y., Arai, H., Uchimoto, Y., et al. (2016). Elucidating the driving force of relaxation of reaction distribution in LiCoO2and LiFePO4Electrodes using X-ray absorption spectroscopy. J. Phys. Chem. C 120, 4739-4743. doi: 10.1021/acs.jpcc.5b10210

Tomandl, I., Vacik, J., Mora Sierra, Y., Granja, C., and Kraus, V. (2017). High resolution imaging of $2 \mathrm{D}$ distribution of lithium in thin samples measured with multipixel detectors in sandwich geometry. Rev. Sci. Instrum. 88:023706. doi: 10.1063/1.4977217

Trask, S. E., Li, Y., Kubal, J. J., Bettge, M., Polzin, B. J., et al. (2014). From coin cells to $400 \mathrm{mAh}$ pouch cells: enhancing performance of high-capacity lithium-ion cells via modifications in electrode constitution and fabrication. J. Power Sourc. 259, 233-244. doi: 10.1016/j.jpowsour.2014.02.077

Ünlü, K., Saglam, M., and Wehring, B. W. (1999). Helium-3 and boron-10 concentration and depth measurements in alloys and semiconductors using NDP. Nucl. Instr. Methods Phys. Res. Sec. A 422, 885-890. doi: 10.1016/S0168-9002(98)01036-5

Utsuro, M., and Ignatovich, V. K. (2010). Reflection, refraction, and transmission of unpolarized neutrons. Handb. Neutron Optic. 31-70. doi: 10.1002/9783527628780.ch2

Vasileiadis, A., de Klerk, N., Smith, R. B., Ganapathy, S. P., Harks, P., et al. (2018). Towards Optimal Performance and In-Depth Understanding of Spinel $\mathrm{Li}_{4} \mathrm{Ti}_{5} \mathrm{O}_{1} 2$ Electrodes Through Phase Field Modeling. Adv. Funct. Materials 28:1705992. doi: 10.1002/adfm.201705992

Villevieille, C. (2015). "Electrochemical characterization of rechargeable lithium batteries," in Rechargeable Lithium Batteries: From Fundamentals to Applications, ed A. A. Franco (Woodhead Publishing), 183-232. doi: 10.1016/B978-1-78242-090-3.00007-9

Wang, C., and Hong, J. (2007). Ionic/electronic conducting characteristics of LiFePO4 cathode materials: the determining factors for high rate performance. Electrochem. Solid-State Lett. 10, A65-A69. doi: 10.1149/1.2409768

Wang, C., Gong, Y., Dai, J., Zhang, L., Xie, H., Pastel, G., et al. (2017). In situ neutron depth profiling of lithium metal-garnet interfaces for solid state batteries. J. Am. Chem. Soc. 139, 14257-14264. doi: 10.1021/jacs.7b07904

Wang, C., Wang, S., Tang, L., Y.-,He, B., Gan, L., et al. (2016). A robust strategy for crafting monodisperse Li4Ti5O12 nanospheres as superior rate anode for lithium ion batteries. Nano Energy 21, 133-144. doi: 10.1016/j.nanoen.2016.01.005

Wang, H., Downing, R. G., Dura, J. A., and Hussey, D. S. (2012). In situ neutron techniques for studying lithium ion batteries. Polymers Energy Storage Deliv. 1096, 91-106. doi: 10.1021/bk-2012-1096.ch006

Wang, J., Liu, D. X., Canova, M., Downing, R. G., Cao, L. R., and Co, A. C. (2014). Profiling lithium distribution in Sn anode for lithiumion batteries with neutrons. J. Radioanal. Nucl. Chem. 301, 277-284. doi: 10.1007/s10967-014-3102-5

Wang, S., Quan, W., Zhu, Z., Yang, Y., Liu, Q., Ren, Y., et al. (2017). Lithium titanate hydrates with superfast and stable cycling in lithium ion batteries. Nat. Commun. 8:627. doi: 10.1038/s41467-017-00574-9

Whitney, S. M., Biegalski, S., R., F., and Downing, G. (2009). Benchmarking and analysis of $6 \mathrm{Li}$ neutron depth profiling of lithium ion cell electrodes. J. Radioanal. Nucl. Chem. 282:173. doi: 10.1007/s10967-009-0229-x

Wilson, W. D., Haggmark, L. G., and Biersack, J. P. (1977). Calculations of nuclear stopping, ranges, and straggling in the low-energy region. Phys. Rev. B 15, 2458-2468. doi: 10.1103/PhysRevB.15.2458

Yu, D. Y. W., Donoue, K., Inoue, T., Fujimoto, M., and Fujitani, S. (2006). Effect of electrode parameters on LiFePO4 cathodes. J. Electrochem. Soc. 153, A835-A839. doi: 10.1149/1.2179199

Yu, Y.-S., Kim, C., Shapiro, D. A., Farmand, M., Qian, D., et al. (2015). Dependence on crystal size of the nanoscale chemical phase distribution and fracture in LixFePO4. Nano Lett. 15, 4282-4288. doi: 10.1021/acs.nanolett.5b01314

Zhang, S. S. (2018). Problem, status, and possible solutions for lithium metal anode of rechargeable batteries. ACS Appl. Energy Mater. 1, 910-920. doi: 10.1021/acsaem.8b00055

Zhang, W., Zhuang, H. L., Fan, L., Gao, L., and Lu, Y. (2018). A cation-anion regulation synergistic anode host for dendrite-free lithium metal batteries. Sci. Adv. 4:2. doi: 10.1126/sciadv.aar4410

Zhang, X., van Hulzen, M., Singh, D. P., Brownrigg, A., Wright, J. P., et al. (2014). Rate-induced solubility and suppression of the first-order phase transition in olivine LiFePO4. Nano Lett. 14, 2279-2285. doi: 10.1021/nl404285y

Zhang, X., Verhallen, T. W., Labohm, F., and Wagemaker, M. (2015). Direct observation of Li-Ion transport in electrodes under nonequilibrium conditions using neutron depth profiling. Adv. Energy Mater. 5:1500498. doi: 10.1002/aenm.201500498

Ziegler, J. F. (1985). The Stopping and Range of Ions in Matter. Boston, MA Springer.

Ziegler, J. F., Cole, G. W., and Baglin, J. E. (1972). Technique for determining concentration profiles of boron impurities in substrates. J. Appl. Phys. 43, 3809-3815. doi: 10.1063/1.1661816

Ziegler, J. F., Ziegler, M. D., and Biersack, J. P. (2010). SRIM - The stopping and range of ions in matter (2010). Nucl. Instr. Methods Phys. Res. Sec. B 268, 1818-1823. doi: 10.1016/j.nimb.2010.02.091

Zuo, X., Zhu, J., Müller-Buschbaum, P., and Cheng, J. (2017). Silicon based lithium-ion battery anodes: A chronicle perspective review. Nano Energy 31, 113-143. doi: 10.1016/j.nanoen.2016.11.013

Conflict of Interest Statement: The authors declare that the research was conducted in the absence of any commercial or financial relationships that could be construed as a potential conflict of interest.

Copyright (c) 2018 Verhallen, Lv and Wagemaker. This is an open-access article distributed under the terms of the Creative Commons Attribution License (CC BY). The use, distribution or reproduction in other forums is permitted, provided the original author(s) and the copyright owner(s) are credited and that the original publication in this journal is cited, in accordance with accepted academic practice. No use, distribution or reproduction is permitted which does not comply with these terms. 